\title{
Quantum distribution functions for radial observables
}

\author{
J. Twamley \\ Theory and Laser Optics Groups \\ Blackett Lab, Imperial College, \\ London, $S W^{\text {r }}$ 2BZ, UK
}

(8 December 1996)

\begin{abstract}
For quantum systems with two dimensional configuration space we construct a physical radial momentum observable. Rescaling the radius we find the dilatonic degrees of freedom form a Weyl algebra. With this we construct the radial Wigner quasi-probability distribution function.
\end{abstract}

The Wigner quasi-probability distribution function is a familiar tool to many working in quantum and atom optics [1]. It is primarily used in the classical-quantum correspondence where the appearance of positive and negative regions of the Wigner function gives easily understood information concerning the probability concentrations and quantum interferences present within the quantum state [2]. Typically one describes the Wigner function on a phase space which is labelled by cartesian coordinates of position and momentum. For physical systems which admit a two dimensional cylindrical symmetry, eg. trapped ultra-cold ions, bose condensates, etc., clearly a polar description of the Wigner function would be more natural. However, no such description has appeared in the literature. In this letter we show, in three stages, how a Wigner function for the radial observables can be constructed. This radial Wigner function could be reconstructed from experimental data much as recent experiments have reconstructed the cartesian Wigner function for the one dimensional motion of a trapped ion [3]. The angular parts of the complete four dimensional Wigner function are complicated by the imposition of singlevaluedness of the wavefunction under a rotation of $2 \pi$ which cause the conjugate angular momentum to become discrete. We leave the angular part for a later work.

The stages towards the construction of a radial-Wigner function proceed as follows: (1) a proper Wigner function possesses marginals which are true probability distributions for the observables whose eigenvalues label the Wigner function and thus the phase space axes. For a single degree of freedom a mere transformation of the cartesian position and momentum into polar form does not yield a proper Wigner function for the polar observables 44. This is also true for higher dimensional phase space representations. Central to the problem is the correct specification of the radial momentum operator. By noting the symmetry action of the momentum on the half-infinite radius observable we construct a physical "conjugate" momentum $\hat{P}^{r} ;(2)$ essential to the construction of the Wigner function is the existence of "point" operators $\hat{A}\left(\lambda_{1}, \lambda_{2}\right)$, which obey, $\operatorname{Tr}\left[\hat{A}\left(\lambda_{1}, \lambda_{2}\right) \hat{A}^{\dagger}\left(\lambda_{1}^{\prime}, \lambda_{2}^{\prime}\right)\right]=\delta\left(\lambda_{1}-\lambda_{1}^{\prime}\right) \delta\left(\lambda_{2}-\lambda_{2}^{\prime}\right)$ [5, 11$]$. We find that the radial $\hat{A}\left(r, P^{r}\right)$, is not completely exponential in the radial position and momentum operators; (3) guided by the form of $\hat{A}\left(r, P^{r}\right)$ we make the coordinate transformation $\hat{v} \equiv \ln \hat{r}$. We find, $\left[\hat{v}, \hat{P}^{r}\right]=i \hbar$, the Weyl algebra. After re-scaling the eigen-basis kets of $\hat{v}$ to recover the standard resolution of unity we can easily construct the radial Wigner function, $W\left(v, P^{r}\right)$. This radial (or dilation) Wigner function gives the proper marginal probability distributions for $\hat{v}$ and $\hat{P}^{r}$. We also note the existence of a dilaton ground state $|0\rangle_{\text {dilations }}$, and calculate the wavefunction of this ground state in the $\hat{r}$ basis. We finally calculate the radial Wigner function for the lowest Schwinger states $|l, 0\rangle$, (these are simultaneous eigenstates of energy and angular momentum), and briefly outline how, given a quantum state in a two dimensional harmonic Fock representation, one can construct the radial reduced density matrix, and from there the radial-Wigner function.

Dirac in his textbook on quantum mechanics [6] introduced the following momentum, conjugate to the radial coordinate

$$
\hat{P}^{D} \equiv \frac{1}{\hat{r}}\left(\hat{x} \hat{P}_{x}+\hat{y} \hat{P}_{y}-i \hbar / 2\right) .
$$

The factor of two difference in the $\hbar$ term arrises because we are working in two dimensions instead of the three in Dirac's case. Using the cartesian commutation relations between $\hat{x}$ and $\hat{P}_{x}$ one can easily confirm that

$$
\left[\hat{r}, \hat{P}^{D}\right]=i \hbar \text {. }
$$

Using the Baker-Campbell-Hausdorff (CBH) expansion and (2) we can show

$$
\exp \left(i \varsigma \hat{P}^{D} / \hbar\right) \hat{r} \exp \left(-i \varsigma \hat{P}^{D} / \hbar\right)=\hat{r}+\varsigma
$$

Since $\hat{r}$ has a half-infinite spectrum, the operator $\hat{P}^{D}$ cannot be self-adjoint. Although this has been noted by many others [7], no self-adjoint radial momentum has been proposed that the author can find. Before going on to construct a self-adjoint radial momentum we first calculate the representation of $\hat{P}^{D}$ in the $\hat{r}$ eigen-ket basis (i.e. $\hat{r}|r\rangle=r|r\rangle)$. From the commutation relation (2) we must have $\left\langle r\left|\hat{P}^{D}\right| \psi\right\rangle \sim-i \hbar\left(\partial_{r}+f(r)\right)\langle r \mid \psi\rangle$. From (11), and expressing the cartesian partial derivatives in polar coordinates we find

$$
\left\langle r\left|\hat{P}^{D}\right| \psi\right\rangle=-i \hbar\left(\partial_{r}+\frac{1}{2 r}\right)\langle r \mid \psi\rangle .
$$

To obtain a self-adjoint momentum operator we must find an operator which respects the half-infinite spectrum of 
$\hat{r}$. This can be achieved if we have $\left[\hat{r}, \hat{P}^{r}\right]=i \hbar \hat{r}$, the Sack dilation algebra [8]. Using the $\mathrm{CBH}$ expansion we can easily show

$$
\exp \left(i \varsigma \hat{P}_{r} / \hbar\right) \hat{r} \exp \left(-i \varsigma \hat{P}_{r} / \hbar\right)=\hat{r} e^{\varsigma} .
$$

To explicitly construct a $\hat{P}^{r}$ we form

$$
\hat{P}^{r} \equiv \hat{r} \hat{P}^{D}-\frac{i \hbar}{2},
$$

where the additional $\hbar / 2$ makes $\hat{P}^{r}$ hermitian. To obtain the representation of $\hat{P}^{r}$ in the $|r\rangle$ basis we simply use (侊) above to find

$$
\left\langle r\left|\hat{P}^{r}\right| \psi\right\rangle=-i \hbar\left(r \partial_{r}+1\right)\langle r \mid \psi\rangle .
$$

From this we can calculate the transition function $\left\langle r \mid P^{r}\right\rangle$ where $\left|P^{r}\right\rangle$ is the eigen-ket of the operator $\hat{P}^{r}$ to be

$$
\left\langle r \mid P^{r}\right\rangle=\frac{1}{\sqrt{2 \pi \hbar}} \frac{r^{i P^{r} / \hbar}}{r} .
$$

This is normalised with the measure $r d r$ and gives

$$
\int_{0}^{\infty} r d r\left\langle r \mid P^{r}\right\rangle\left\langle P^{\prime r} \mid r\right\rangle=\delta\left(P^{r}-P^{\prime r}\right) .
$$

The action of $\hat{r}$ on $\hat{P}^{r}$ is not a simple scaling, instead

$$
e^{-i \varsigma \hat{r} / \hbar} \hat{P}^{r} e^{i \varsigma \hat{r} / \hbar}=\hat{P}^{r}+\varsigma \hat{r} .
$$

To form a displacement, or point, operator we might be tempted to exponentiate a linear combination of the position and momentum operators in analogy with the harmonic oscillator. However this construction does not result in a unique adjoint action of the displacement operator on $\hat{r}$ and $\hat{P}^{r}$. From [10] we see

$$
\begin{aligned}
\mathcal{D}(a, m) & \equiv \exp \left(\frac{i a}{\hbar}\left[\hat{P}^{r}+m \hat{r}\right]\right) \\
& =\exp \left(\frac{i a}{\hbar} \hat{P}^{r}\right) \exp \left[\frac{i m \hat{r}}{\hbar}\left(1-e^{-a}\right)\right] \\
& =\exp \left[\frac{i m \hat{r}}{\hbar}\left(e^{a}-1\right)\right] \exp \left(\frac{i a}{\hbar} \hat{P}^{r}\right) .
\end{aligned}
$$

Thus the choice of ordering alters the action of $\mathcal{D}(a, m)$ on the operators $\hat{r}$ and $\hat{P}^{r}$. This is to be expected since the algebra is not symmetric in $\hat{r}$ and $\hat{P}^{r}$. One must explicitly specify the ordering in this displacement operator as is done in most treatments of displacement operators for spin systems.

An essential property for a displacement operator is [5, 11],

$$
\operatorname{Tr}\left[D(\lambda, \mu) D^{\dagger}\left(\lambda^{\prime}, \mu^{\prime}\right)\right] \approx \delta\left(\lambda-\lambda^{\prime}\right) \delta\left(\mu-\mu^{\prime}\right) .
$$

No operator in the form of products of exponentials of $\hat{r}$ and $\hat{P}^{r}$ will satisfy (9). We instead consider the operator [12],

$$
D(\lambda, \mu) \equiv \exp \left(i \mu \hat{P}^{r} / 2 \hbar\right) r^{i \lambda} \exp \left(i \mu \hat{P}^{r} / 2 \hbar\right),
$$

where $\lambda \in[0,+\infty)$. Taking the trace over $|r\rangle$ with measure $\frac{r d r}{2 \pi}$, from $r=0$ to $r=+\infty$ gives

$$
\operatorname{Tr}\left[D(\lambda, \mu) D^{\dagger}\left(\lambda^{\prime}, \mu^{\prime}\right)\right]=\frac{1}{e^{2 \mu}} \delta\left(\lambda-\lambda^{\prime}\right) \delta\left(\mu-\mu^{\prime}\right) .
$$

One can show $D^{\dagger}(\lambda, \mu) \hat{r} D(\lambda, \mu)=\hat{r} e^{-\mu}$. However to evaluate $D^{\dagger}(\lambda, \mu) \hat{P}^{r} D(\lambda, \mu)$ one must calculate $\hat{r}^{\alpha} \hat{P}^{r} \hat{r}^{-\alpha}$. This is done by expressing $\hat{P}^{r}$ in terms of $\hat{P}^{D}$ through (6), using the simpler commutation relations for $\hat{P}^{D}$, the rule $\left[f(\hat{r}), \hat{P}^{D}\right]=i \hbar \partial f(\hat{r}) / \partial \hat{r}$, and finally transforming back to $\hat{P}^{r}$. This surprisingly gives $D^{\dagger}(\lambda, \mu) \hat{P}^{r} D(\lambda, \mu)=$ $\hat{P}^{r}+\lambda$.

Thus, by using the new "displacement" operator (10), one can (except for the exponential factor in (11)), almost completely regain the properties of the Harmonic oscillator displacement operator. Although one could now proceed to construct a Wigner function with this displacement operator, the replacement of $\exp (i \lambda \hat{r})$ with $\hat{r}^{i \lambda}$ in (10), and the exponential prefactor in (11) point towards a clearer understanding of the situation. Guided by these signals we now make the transformation to a new coordinate operator,

$$
\hat{v} \equiv \ln \hat{r} .
$$

Using the definition of $\hat{P}^{r}$ in terms of $\hat{P}^{D}$ (6), we can show

$$
\left[\hat{v}, \hat{P}^{r}\right]=\left[\ln \hat{r}, \hat{P}^{r}\right]=\left[\ln \hat{r}, \hat{r} \hat{P}^{D}\right]=\hat{r}\left[\ln \hat{r}, \hat{P}^{D}\right]=\hat{r} \frac{i \hbar}{\hat{r}}=i \hbar
$$

With this we have re-gained the algebra of the original cartesian position and momentum observables. Since the spectrum for both $\hat{v}$ and $\hat{P}^{r}$ ranges from $(-\infty,+\infty)$ the translation generating Weyl algebra in (13) properly respects the spectrum of both operators with the result that both $\hat{v}$ and $\hat{P}^{r}$ are self-adjoint and represent physical observables. We can construct creation and annihilation operators in the same manner as the harmonic oscillator, $\hat{a} \equiv\left(\hat{v}+i \hat{P}^{r}\right) / \sqrt{2}$, and $\hat{a}^{\dagger} \equiv\left(\hat{v}-i \hat{P}^{r}\right) / \sqrt{2}$. In particular, there exists a proper vacuum state $|0\rangle_{\text {dilations }}$ which is annihilated by $\hat{a}$.

Not everything is straightforward however. The resolution of unity in the $|r\rangle$ basis becomes, in the eigenbasis of $\hat{v}$,

$$
\begin{aligned}
2 \pi \mathbb{1} & =\int_{-\infty}^{+\infty} d x|x\rangle\left\langle x\left|=\int_{0}^{\infty} r d r\right| r\right\rangle\langle r| \\
& =\int_{-\infty}^{+\infty} e^{2 v} d v|v\rangle\langle v| .
\end{aligned}
$$

With this resolution of unity we must have 


$$
\mathbb{1}\left|v^{\prime}\right\rangle=\left|v^{\prime}\right\rangle=\frac{1}{2 \pi} \int_{-\infty}^{+\infty} e^{2 v} d v|v\rangle\left\langle v \mid v^{\prime}\right\rangle,
$$

and thus $\left\langle v \mid v^{\prime}\right\rangle \equiv \frac{1}{e^{2 v}} \delta\left(v-v^{\prime}\right)=2 \delta\left(e^{2 v}-e^{2 v^{\prime}}\right)$. This exponential measure complicates matters and so to simplify we rescale the basis kets to be

$$
|\bar{v}\rangle^{\bullet} \equiv e^{\bar{v}}\left|e^{\bar{v}}\right\rangle_{r}
$$

where $\left|e^{\bar{v}}\right\rangle_{r}$ is a eigenket of $\hat{r}$ with eigenvalue $e^{\bar{v}}$. The resolution of the identity in the new $|\bar{v}\rangle^{\bullet}$ basis is, $2 \pi \mathbb{1}=$ $\int_{-\infty}^{+\infty} d \bar{v}|\bar{v}\rangle^{\bullet \bullet}\langle\bar{v}|$. The inner product between $|\bar{v}\rangle^{\bullet}$ basis kets is now simply $\bullet\langle\bar{v}| \bar{v}^{\prime} \bullet^{\bullet} \equiv \delta\left(\bar{v}-\bar{v}^{\prime}\right)$. This rescaling will primarily appear in the calculation of $\bullet\langle\bar{v} \mid \psi\rangle$ when we have $\langle r \mid \psi\rangle$. To be certain that everything is identical to the Harmonic oscillator case we finally evaluate $\bullet\left\langle\bar{v}\left|\hat{P}^{r}\right| \psi\right\rangle$. Since $|\bar{v}\rangle^{\bullet}=r|r\rangle$ where $r=e^{\bar{v}}$, we have

$$
\begin{aligned}
& \bullet\left\langle\bar{v}\left|\hat{P}^{r}\right| \psi\right\rangle=\left.r\left\langle r\left|\hat{P}^{r}\right| \psi\right\rangle\right|_{r=e^{\bar{v}}}=\left.r\left[-i \hbar\left(r \partial_{r}+1\right)\right]\langle r \mid \psi\rangle\right|_{r=e^{\bar{v}}} \\
& \quad=\left.r\left[-i \hbar\left(r \partial_{r}+1\right) \frac{1}{r}\right] \bullet\langle\bar{v} \mid \psi\rangle\right|_{r=e^{\bar{v}}} \\
& \quad=-\left.i \hbar r \partial_{r} \bullet\langle\bar{v} \mid \psi\rangle\right|_{r=e^{\bar{v}}}=-i \hbar \partial_{\bar{v}}^{\bullet}\langle\bar{v} \mid \psi\rangle .
\end{aligned}
$$

We see that the momentum operator $\hat{P}^{r}$, in the basis $|\bar{v}\rangle^{\bullet}$ is the familiar $-i \hbar \partial_{\bar{v}}$, the same as the cartesian case.

As in the Harmonic oscillator case we define the Wigner and s-ordered quasi-distribution function to be

$$
W(\xi, s) \equiv \int \frac{d^{2} \alpha}{\pi} e^{\alpha \xi^{*}-\alpha^{*} \xi} C(\alpha, s)
$$

where $C(\alpha, s)$ is the s-ordered displacement operator

$$
C(\alpha, s) \equiv \operatorname{Tr}\left[\rho D(\alpha) e^{s|\alpha|^{2} / 2}\right],
$$

and $D(\alpha) \equiv \exp \left(\alpha \hat{a}^{\dagger}-\alpha^{*} \hat{a}\right)$, where $\hat{a}^{\dagger}$ and $\hat{a}$, the dilatonic creation and annihilation operators, are defined as above. For $s=0, \xi=\gamma+i \delta$, the Wigner function becomes

$$
W(\gamma, \delta)=\frac{1}{2 \pi} \int d \varepsilon e^{-i \varepsilon \delta \bullet}\left\langle\gamma+\frac{\varepsilon}{2}|\rho| \gamma-\frac{\varepsilon}{2}\right\rangle \bullet .
$$

We now calculate the Wigner function for the quantum states $\rho_{l} \equiv|l, 0\rangle\langle l, 0|$, where $|l, m\rangle$ is the Schwinger angu(hal momentum state given by

$$
|l, m\rangle \equiv \frac{1}{\sqrt{(l+m) !(l-m) !}} \hat{A}_{+}^{\dagger(l+m)} \hat{A}_{-}^{\dagger(l-m)}|0,0\rangle
$$

where $\hat{A}_{+} \equiv\left(\hat{a}_{x}-i \hat{a}_{y}\right) / \sqrt{2}, \hat{A}_{-} \equiv\left(\hat{a}_{x}+i \hat{a}_{y}\right) / \sqrt{2}[1]$. Either by solving the generating differential equation (as is done in 13.) or by solving the harmonic oscillator radial equation one finds $\langle r, \phi \mid l, m\rangle=R_{l, m}(r) \Theta_{m}(\phi)$, where

$$
\begin{aligned}
R_{l, m}(r) & =\beta \sqrt{\frac{2(l-|m|) !}{(l+|m|) !}}(\beta r)^{2|m|} e^{-\beta^{2} r^{2} / 2} \mathrm{~L}_{l-|m|}^{2|m|}\left(\beta^{2} r^{2}\right)(-1)^{l-|m|} \\
\Theta_{m}(\phi) & =\frac{1}{\sqrt{2 \pi}} e^{2 i m \phi}
\end{aligned}
$$

$L_{n}^{\alpha}$ is the associated Laguerre polynomial and $\beta=1 / \sqrt{\hbar}$. Integrating out the $\phi$ dependence, setting $\hbar=1$ and using (17) we obtain

$$
\bullet\langle\bar{v} \mid l, 0\rangle=\sqrt{2} e^{\bar{v}} e^{-e^{2 \bar{v}} / 2} \mathrm{~L}_{l}^{0}\left(e^{2 \bar{v}}\right)(-1)^{l} .
$$

Inserting (25) into (21) we finally get

$$
W_{l}(\gamma, \delta)=\frac{2 e^{2 \gamma}}{\pi} \int_{-\infty}^{+\infty} d \varepsilon e^{-2 i \varepsilon \delta} \exp \left[-e^{2 \gamma} \cosh 2 \varepsilon\right] \mathrm{L}_{l}\left(e^{2(\gamma+\varepsilon)}\right) \mathrm{L}_{l}\left(e^{2(\gamma-\varepsilon)}\right) .
$$

The radial Wigner function, $(26)$, is numerically integrated for the first four $|l, 0\rangle$ states and plotted in Figs. 1(a)-1(d). The presence of negative regions in these Wigner functions is not surprising as the pure states $|l, 0\rangle$ are reminiscent of Fock states. One can construct the dilatonic coherent states $|\alpha\rangle \equiv D(\alpha)|0\rangle_{\text {dilatons }}$. More interesting is $\langle r \mid 0\rangle_{\text {dilatons. From the definition }}$ $\hat{a}|0\rangle_{\text {dilatons }}=0$, and $\sqrt{2} \hat{a}=\hat{v}+i \hat{P}^{r}$, we can evaluate $\bullet\langle\bar{v}|\hat{a}| 0\rangle_{\text {dilatons }}=0$ to get $\bullet\langle\bar{v} \mid 0\rangle_{\text {dilatons }}=N \exp \left(-\bar{v}^{2} / 2\right)$, where $N^{2}=\sqrt{\pi}$. Again using (17) we obtain

$$
\langle r \mid 0\rangle_{\text {dilatons }}=\frac{1}{r \sqrt{\pi}} \exp \left(-\frac{1}{2} \ln r \ln r\right)=\frac{r^{-\frac{1}{2} \ln r}}{r \sqrt{\pi}},
$$

which is normalised to unity, $\int_{0}^{+\infty} r d r\left|\langle r \mid 0\rangle_{\text {dilatons }}\right|^{2}=$ 1 .

In this letter we examined the problem of constructing a Wigner function for the two dimensional radial subspace of a quantum system possessing two continuous 
degrees of freedom (or a four dimensional Wigner function). Since the radial subspace is labelled by an operator with an half-infinite spectrum, previous attempts to define a physical conjugate momentum have failed. By choosing a momentum operator which respected the spectrum of the radial coordinate we found we could construct a physically meaningful radial conjugate momentum. With a logarithmic relabelling of the radial coordinate the new coordinate and momentum satisfied a Weyl algebra and we were thus able to carry over all the techniques associated with the harmonic oscillator to construct a Wigner function. One can identify a ground state for these observables which is destroyed by a dilatonic annihilation operator. We finally examined the radial-Wigner function for particular quantum states and found the radial wavefunction for the dilatonic vacuum state. To examine the radial-Wigner function for more general quantum states is difficult. If one has the density matrix in a $x-y$ Fock state basis, one must first transform $\rho$ into the Schwinger basis of angular momentum kets, $\rho=\sum C_{l m ; l^{\prime} m^{\prime}}|l, m\rangle\left\langle l^{\prime}, m^{\prime}\right|$. One can then find $\left\langle r, \phi|\rho| r^{\prime}, \phi^{\prime}\right\rangle$. Finally one must trace over $\phi$. However, even though we have traced out over $\phi$ one will still end up with a sum over $m$. This is so because, in contrast with the cartesian Fock state decomposition, the radial wavefunction is labelled by both quantum numbers $l$ and $m$ while the angular part is only labelled by $m$. We also note that the Wigner function here gives the correct marginals for the operators $\hat{P}^{r}$ and $\hat{v}$. Once these marginals and their corresponding integration measures are obtained one can rescale $v$ to $r$. One cannot rescale the axis in Fig. 1 to find the Wigner function for $\hat{P}^{r}$ and $\hat{r}$ without altering the integration measure and thus, the function form of the Wigner function. Finally, although an observable, the radial operator $\hat{v}=\ln \hat{r}$, may not be easy to measure directly. However, its should be possible, through standard reconstruction techniques, to numerically approximate the radial Wigner function from experimental data.

The author thanks V. Bužek of Imperial College, London, UK and A. Hurst at the University of Adelaide, South Australia, for enlightening discussions. This work was supported through a European Human Capital and Mobility Fellowship.

[1] M. Hillery, R. F. O' Connell, M. O. Scully, and E. P. Wigner, Phys. Rep. 106, 121 (1984).

[2] J. P. Dowling, W. P. Schleich, and J. A. Wheeler, Ann. Phys. 7, 423 (1991).

[3] D. Leibfried, D. M. Meekhof, B. E. King, C. Monroe, W. M. Itano, and D. J. Wineland, Phys. Rev. 77, 4281 (1996).

[4] B. M. Garraway and P. L. Knight, Phys. Rev. A 46,
R5346 (1992).

[5] W. K. Wootters, Ann. Phys. (N.Y.) 176, 1 (1987); J. A. Vaccaro, Opt. Commun. 113, 421 (1995); T. Opatrný, D. G. Welsch, and V. Bužek, Phys. Rev. A 53, 3822 (1996).

[6] P. A. M. Dirac, Quantum Mechanics, Section 38, (Oxford University Press, 1958).

[7] A. Messiah, Quantum Mechanics I, Chapter IX section 2, (North-Holland, Amsterdam, 1965); R. L. Libhoff, I. Nebenzahl, and H. H. Fleischmann, Am. J. Phys. 41, 976 (1973); P. D. Robinson and J. O. Hirschfelder, J. Math. Phys. 4, 338 (1963); O. L. De Lange and R. E. Raab, Operator Methods in Quantum Mechanics, (Clarendon Press, Oxford, 1991).

[8] R. A. Sack, Phil. Mag. 3, 497 (1958).

[9] T. Opatrný, V. Bužek, J. Bajer, and G. Drobný, Phys. Rev. A 52, 2419 (1995); M. O. Scully and K. Wódkiewicz, Found. Phys. 24, 85 (1994).

[10] W. Witschel, J. Phys. A 7, 1847 (1974).

[11] K. E. Cahill and R. J. Glauber, Phys. Rev. 177, 1857 (1969); 177, 1882 (1969).

[12] W. H. Lousiell, Quantum Statistical Properties of Radiation, (Wiley-Interscience, New York, 1990), equ. 3.4.36.

[13] C. Cohen-Tannoudji, B. Diu, and F. Lalöe, Quantum Mechanics I, (J. Wiley and Sons, NY, 1977), Complement DVId.

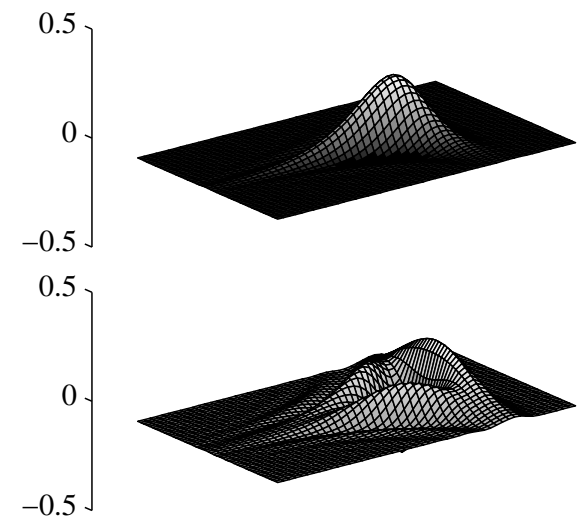

(a)

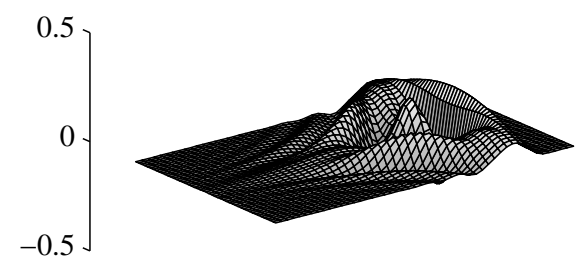

(c)

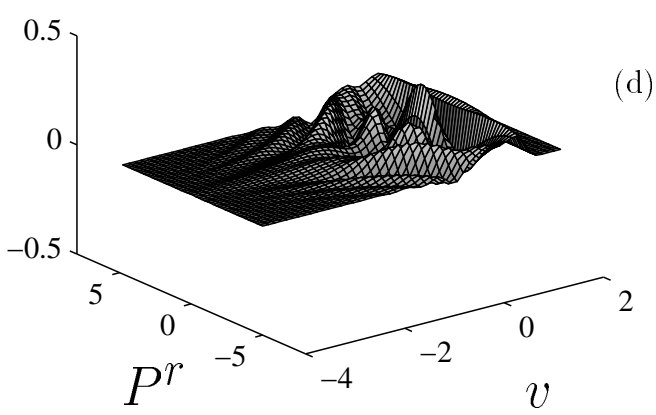

FIG. 1. (a)-(d) Radial Wigner Functions for the states $|l, 0\rangle, l=0, . ., 3$. 